\title{
Aluminum Nitride piezo-MEMS on polyimide flexible substrates
}

\author{
Simona Petroni ${ }^{\mathrm{a}, *}$, Carola La Tegola $^{\mathrm{b}}$, Giuseppe Caretto ${ }^{\mathrm{b}}$, Adriana Campa ${ }^{\mathrm{b}}$, Adriana Passaseo ${ }^{\mathrm{b}}$, \\ Massimo De Vittorio ${ }^{\mathrm{a}, \mathrm{c}}$, Roberto Cingolani ${ }^{\mathrm{d}}$ \\ a IIT-Italian Institute of Technology, Via Barsanti sn, 73010 Arnesano, Lecce, Italy \\ ${ }^{\mathrm{b}}$ CNR NANO - Institute of Nanoscience, Via Arnesano, 73100 Lecce, Italy \\ ${ }^{\mathrm{C}}$ University of Salento, Via per Arnesano, Lecce 73100, Italy \\ ${ }^{\mathrm{d}}$ IIT-Italian Institute of Technology, Via Morego, 30, 16163 Genoa, Italy
}

\section{A R T I C L E I N F}

\section{Article history}

Available online 25 February 2011

\section{Keywords:}

AlN

Polyimide

Flexible substrates

Piezoelectric

MEMS

\begin{abstract}
A B S T R A C T
In this work the micro-fabrication of flexible MicroElectroMechanical transducers based on the piezoelectric effect is reported. We developed the technological protocol to realize a piezoelectric transducer composed by a Molybdenum (Mo) top electrode, the Aluminum Nitride active layer and a Mo bottom electrode on a polymeric tape. The process starts from the DC sputtering deposition of the Molybdenum layer at room temperature on Kapton HN. The Molybdenum is chosen not only for its electrical properties but also because it enhances the crystal orientation of AlN. The next step is the deposition of AIN that occurs at high temperature, around $250^{\circ} \mathrm{C}$. Temperature and physical sputtering enhanced by applying a DC bias on the substrates are two important parameters to improve the crystal orientation of the film. These extreme growth conditions guarantee a very good crystal structure without damaging the Kapton substrate. Then a final Mo layer is sputtered at room temperature. SU8-25 thick photo resist is used to define the top electrode and the AIN layer, and in a second mask step the Mo bottom electrode. The developer, the PG remover and SU8 negative resist itself have shown a chemical compatibility with Kapton HN. We measured the piezoelectric response on a capacitor test structure: through the Dynamic Mechanical Analyzer we applied controlled forces, and at the same time, by an LCR meter we performed measurements of the capacitance.
\end{abstract}

(c) 2011 Elsevier B.V. All rights reserved.

\section{Introduction}

Polymers and soft materials have big advantages compared to classical semiconductor materials in terms of cost, flexible packaging, roll to roll large scale fabrication and biocompatibility. On the other hand the reliability of polymers is still very low to guarantee good performances on millions of operating cycles. The study of soft MEMS devices captured great interest in the last few decades [1] and the development of new micro-fabrication processes represents a big part of this study [2-4]. Between the classical microfabrication processes the deposition by sputtering does not show, in principle, constrains on the choice of the substrate. For instance a natural piezoelectric material as Aluminum Nitride could be grown by sputtering in highly oriented polycrystalline phase [5] at relatively low temperature $\left(\sim 300^{\circ} \mathrm{C}\right)$ compatible with CMOS technology and to soft substrates such as Kapton polymer. In our work we integrate the flexible polymer, Kapton HN, having proved reliability performances on large scale production, with an Aluminum Nitride thin film deposited by sputtering technique. Kapton

\footnotetext{
* Corresponding author. Tel.: +390 832 295726; fax: +39 0832295708.

E-mail addresses: simona.petroni@iit.it, simopetroni@gmail.com (S. Petroni).
}

and AlN are widely used in MEMS applications. Kapton HN is used in all the applications requiring polyimide films with an excellent balance of electrical and mechanical properties over a wide temperature range $\left(-269^{\circ} \mathrm{C}\right.$ to $\left.400^{\circ} \mathrm{C}\right)$, whereas Aluminum Nitride is a material of great technological interest for its wide band gap, high electrical resistivity, high thermal conductivity, high break down voltage and good piezoelectricity. AlN is very common in MEMS and in acoustic applications such as bulk acoustic wave (BAW) [6] and surface acoustic wave (SAW) resonators and filters [7].

\section{Soft MEMS fabrication}

The Kapton tape used for our devices is composed by $25 \mu \mathrm{m}$ of polyimide and $60 \mu \mathrm{m}$ of silicone adhesives (H-old SpA). The tape has been laminated on a silicon support and cleaned with Acetone, IPA and DI water, then dried with a nitrogen flow.

On this samples the DC sputtering deposition of Molybdenum bottom electrode, Aluminum Nitride piezoelectric layer and Mo top electrode is performed in a single run with a LLSEVO production tool. This tool can work on very large areas, 9 holders for $8^{\prime \prime}$ wafers are present on a cylindrical cage that rotates around its axis 


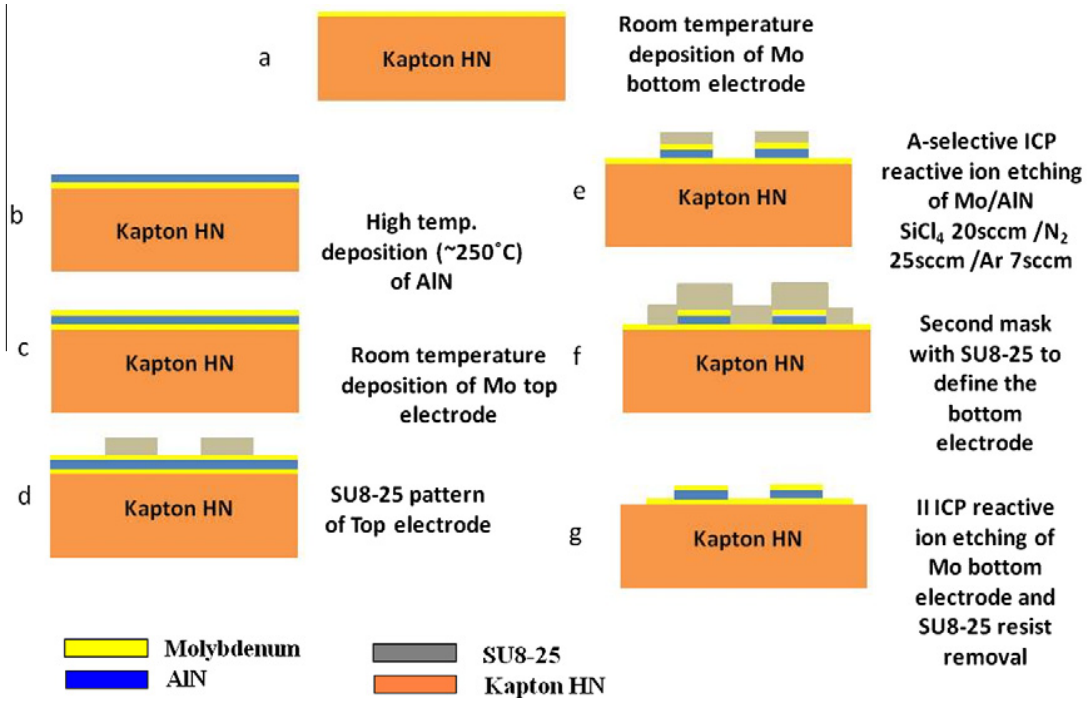

Fig. 1. Process flow for the fabrication of the flexible transducers.

to guarantee an uniformity $<2 \%$. The Mo layers have been sputtered at room temperature and low power, whereas the AlN layer at $250^{\circ} \mathrm{C}$ and with a high energy sputtering, promoted by applying a bias voltage of $-50 \mathrm{~V}$ to the substrate.

After the deposition, the sample is spin coated with negative resist SU8-25; by a spinning speed of $5000 \mathrm{rpm}$ for $30 \mathrm{~s}$ a resist thickness around $10 \mu \mathrm{m}$ has been obtained. The pre-exposure bake lasts $2 \mathrm{~min}$ at $65^{\circ} \mathrm{C}$ and $4 \mathrm{~min}$ at $95^{\circ} \mathrm{C}$. The resist is exposed in soft contact mode for $25 \mathrm{~s}$ on a KarlSuss Mask aligner with lamp power density of $20 \mathrm{~mW} / \mathrm{cm}^{2}$. After the exposure, the above reported bake is repeated.

The top electrode and the piezoelectric layer are defined in a single etching step with the same shape through an ICP (Inductively Coupled Plasma) process having the following parameters: $\mathrm{SiCl}_{4} 20 \mathrm{sccm} / \mathrm{N}_{2} 25 \mathrm{sccm} / \mathrm{Ar} 7 \mathrm{sccm}$, the power applied to the platen is 45 Watt and to the coil is 100 Watt. This etching is not selective and the time is set according to the thickness of the layer, with a slight over-etch through the Mo at the bottom. We have observed that in our samples the thickness of AlN must be higher than $700 \mathrm{~nm}$ in order to have a good electrical isolation between top and bottom electrode. Moreover, the isolation of the devices on the Kapton substrate, avoids short-circuit between top and bottom electrodes during the slicing process.

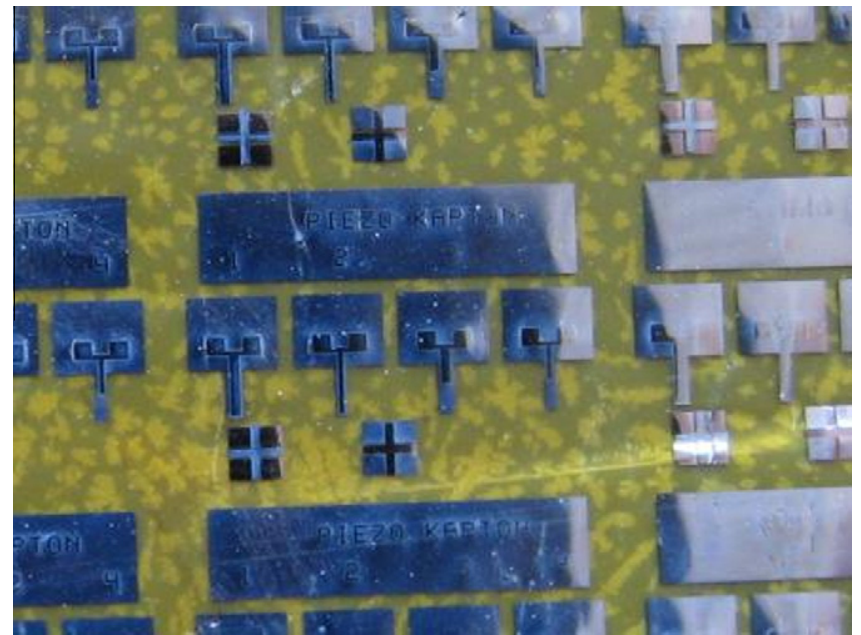

Fig. 2. Flexible beam structures realized on Kapton.
After the etching of the top electrode and the active layer shown in the step (e) of Fig. 1, the resist SU8 is left to protect the top electrode and a second SU8-25 masking process is performed in the step (f) with the same conditions reported above. In the step ( $\mathrm{g}$ ) we dry etch the Molybdenum to define the bottom electrode on Kapton. To complete the device fabrication the SU8-25 is removed dipping the sample into hot PG remover $\left(120^{\circ} \mathrm{C}\right)$, then into water and IPA. This process is very simple and robust, it allows to fabricate flexible transducers on large scale at very low cost.

The classical MEMS approach to uptake free standing structures for sensing and actuation, was exploited for the fabrication of cantilevers on Kapton (Fig. 2) composed by a Molybdenum bottom electrode, the AlN active layer and a Mo top electrode [8-10].

The contact pads of the top electrode are designed in the configuration GSG (Ground-Signal-Ground) with a pitch of $150 \mu \mathrm{m}$ for further RF analysis by Network Vector Analyzer, to determinate the resonant frequency of the beam .

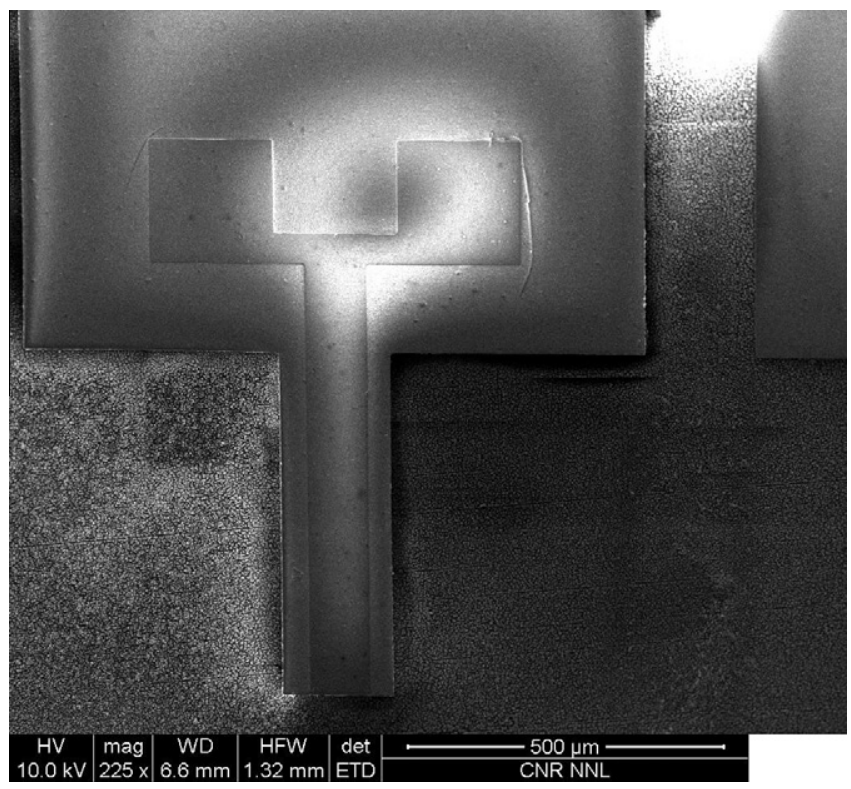

Fig. 3. SEM image of the piezoelectric beam. Width of the top electrode is $100 \mu \mathrm{m}$, whereas the bottom electrode is $180 \mu \mathrm{m}$ wide. 
To release the beam we dry etched the Kapton with a simple plasma oxygen, we used the ICP and set the following parameters: pressure $45 \mathrm{mtorr}, \mathrm{O}_{2}$ flow $45 \mathrm{sccm}$, RF power of $600 \mathrm{~W}$ applied to the cold platen and power of $200 \mathrm{~W}$ to the coil.

We extended the etching time as far as 20 min to release the beam. The etching rate on Kapton for this recipe is around $0,78 \mu \mathrm{m} / \mathrm{min}$, resulting in an undercut of less than $16 \mu \mathrm{m}$. This is not enough to release the beam, taking into account that the width of the cantilever is $180 \mu \mathrm{m}$. In Fig. 3 the SEM image of the piezoelectric beam after the oxygen plasma is shown; here it is possible to notice the undercut of the Kapton. The fabrication of free standing structures through the dry etching is still under development.

\section{Results and analysis}

The materials grown on Kapton have been characterized on a X-Pert PRO MRD diffractometer (Panalytical). The results shown in Fig. 4 present two very strong peaks: the first at $\theta$ about $36^{\circ}$ is due to the reflections $\left(\begin{array}{lll}0 & 0 & 0\end{array}\right)$ of $\mathrm{AlN}$ and the second around 41 is due to the reflections ( 110 ) of Molybdenum. The full width at half maximum (FWHM) of the rocking curves is $0.54^{\circ}$ for the AlN and $0.52^{\circ}$ for the Mo. Narrow peaks confirm the $c$-axis highly oriented texture of piezoelectric layer and it is very important to enhance the piezoelectric coefficient of the material [5]. This result can be compared to the FWHM of $9.1^{\circ}$ reported by Akiyama et al. in which the AlN is grown at room temperature on Copper [11].

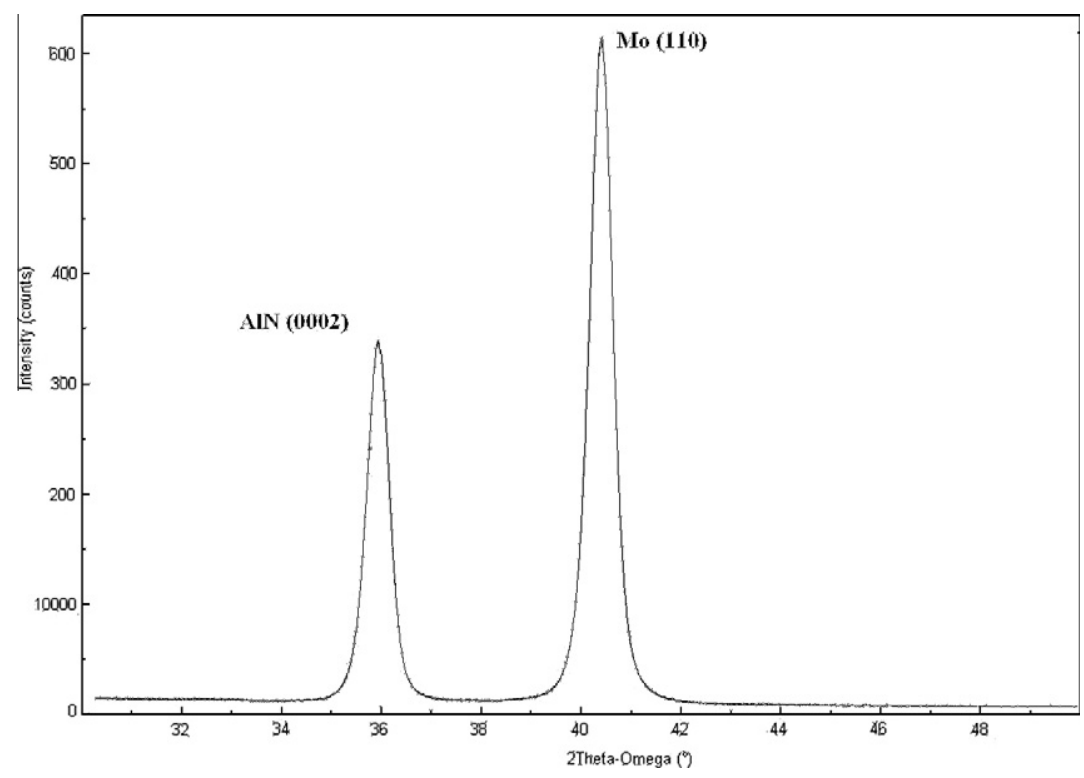

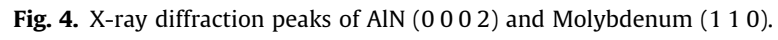

(b)

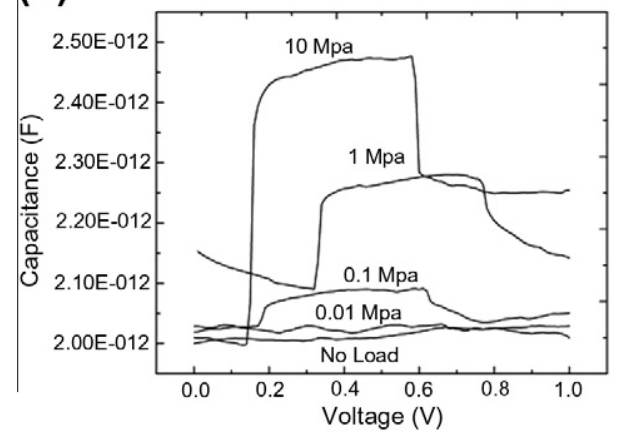

(a)

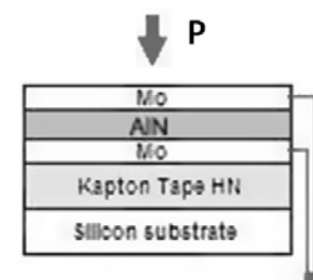

(c)
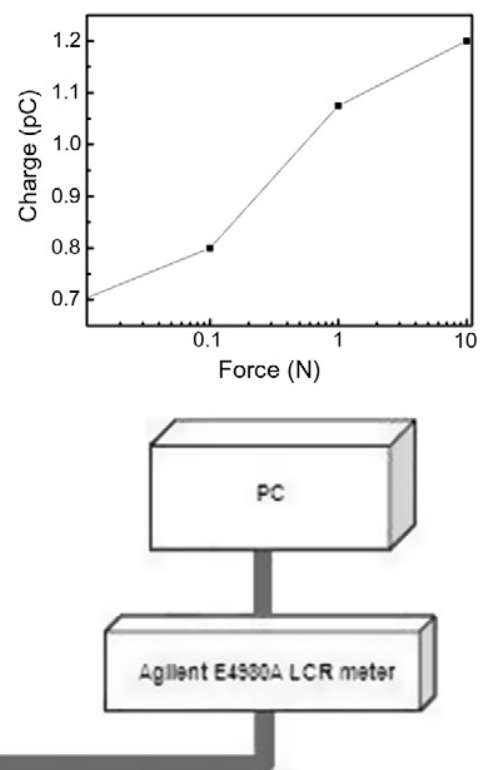

Fig. 5. Measure set up (a) measure of capacitance under the applied pressures from 0.01 to $10 \mathrm{MPa}$ (b) charge corresponding to each force applied. 
The morphology of the Mo surface after the sputtering deposition of the entire stack was characterize by the Atomic Force Microscopy, the roughness on the $5 \mu \mathrm{m}$ scan is $3.6 \mathrm{~nm}$ and the layer under the optic microscope is uniform and cracks free.

In order to test the piezoelectric response of the AIN under stress, we measured the capacitance between the two Mo electrodes of a capacitor at $1 \mathrm{MHz}$ under a voltage raising from 0 to $1 \mathrm{~V}$ with a step bias of $0.01 \mathrm{~V}$, through the set up showed in Fig. 5a. After some seconds a controlled pressure was applied (Fig. 5b) on the structure producing an instantaneous increasing of the capacitance due to the charge generated under stress. The pressures applied are the following: $0.01,0.1,1$, and $10 \mathrm{MPa}$, for each of them we observed a signal and the charge generated was determinate integrating the area under the $C-V$ curve (Fig $5 c$ ).

\section{Conclusions}

The fabrication of soft MEMS that can be a part of a flexible pressure sensors for synthetic skin represents a big challenge for the realization of biomimetic prosthesis and robotic parts. We face this challenge developing a flexible piezoelectric transducer, whose structure is very far from the engineered EAP (electro-active polymers). EAP practical application in robotic systems [12] is still limited by low actuation force, low mechanical energy density, and low robustness, whereas our approach, based on simple and robust piezoelectric transducers, on soft substrates, overcomes these limits. The fabricated devices, exploit the integration of a good piezoelectric material, AlN, with a soft substrates, namely Kapton, both exhibiting excellent mechanical and electrical properties in a wide range of temperature.

\section{References}

[1] C. Liu, Advanced Materials 19 (2007) 3783-3790.

[2] Y. Qi, N.T. Jafferis, K. Lyons, C.M. Lee, H. Ahmad, M.C. McAlpine, Nano Letters 10 (2010) 524-528.

[3] M. Akiyama, Y. Morofuji, T. Kamohara, K. Nishikubo, Y. Ooishi, Advanced Functional Materials 17 (2007) 458-462.

[4] M. Tsubai, N. Ueno, O. Fukuda, SICE-ICASE International Joint Conference (2006) 3227-3830.

[5] M.A. Dubois, P. Muralt, Journal of Applied Physics 89 (2001) 6389-6395.

[6] H.H. Kim, B.K. Ju, Y.H. Lee, S.H. Lee, J.K. Lee, S.W. Kim, Sensors and Actuators A 89 (2001) 255-258.

[7] I. Ingrosso, S. Petroni, D. Altamura, M. De Vittorio, C. Combi, A. Passaseo, Microelectronic Engineering 84 (2007) 1320-1324.

[8] J. Olivares, E. Iborra, M. Clement, L. Vergara, J. Sangrador, A. Sanz-Hervas, Sensors and Actuators A 123-124 (2005) 590-595.

[9] H. Lee, J. Park, K. Lee, Y. Ko, J. Bu, Integrated Ferroelectrics 69 (2005) 323-332.

[10] L.P. Wang, E. Ginsburg, F. Gerfers, D. Samaria-Rubio, B. Weinfeld, Q. Ma, V. Rao, IEEE Sensors (2006) 10-13.

[11] M. Akiyama, Y. Morofuji, K. Nishikubo, T. Kamohara, Applied Physics Letters 92 (2008) 043509

[12] K.J. Kim, S. Tadokoro, Artificial Muscles and Sensors, Springer-Verlag London Limited, 2007. 